\title{
Control of Cauchy Problem for a Laplacian Operator
}

\author{
Sadou Tao \\ Department of Mathematics, Université Joseph KI-ZERBO, Ouagadougou, Burkina Faso
}

Email address:

sadoutao.tao9@gmail.com

\section{To cite this article:}

Sadou Tao. Control of Cauchy Problem for a Laplacian Operator. American Journal of Applied Mathematics. Special Issue: Numerical Analysis and Control Theory. Vol. 8, No. 5, 2020, pp. 271-277. DOI: 10.11648/j.ajam.20200805.15

Received: May 18, 2020; Accepted: August 25, 2020; Published: September 21, 2020

\begin{abstract}
In this paper we study the control of an ill-posed system relating to the Cauchy problem for an elliptical operator. The control of Cauchy systems for an elliptical operator has already been studied by many authors. But it still seems to be globally an open problem. Of all the studies that have been done on this problem, it is assumed that the set of admissible couple-state must be nonempty to make sense of the problem. This is the case of J. L. Lions in [6] who gave various examples of the admissible set to make a sense of the problem. O. Nakoulima in [9] uses the regularization-penalization method to approach the problem by a sequence of well-posed control problems, he obtains the convergence of the processus in a particular case of the admissible set. G. Mophou and O. Nakoulima in [10] do the same study and obtain the convergence of the processus when the interior of the admissible set is non empty. In this work, we give an approximate solution without an additional condition on the set of admissible couple-state. We propose a method which consists in associating with the singular control problem a "family" of controls of well posed problems. We propose as an alternative the stackelberg control which is a multiple-objective optimization approach proposed by H. Von Stackelberg in [12].
\end{abstract}

Keywords: Systems Governed by PDEs, Stackelberg Control, Cauchy Problem, Cost Function

\section{Statement of the Problem}

Let $\Omega$ be an open bounded subset of $R^{n}$, with a boundary $\Gamma$ of class $\mathrm{C}^{2}$.

$\Gamma=\Gamma_{e} \cup \Gamma_{i}$ with $\Gamma_{e} \cap \Gamma_{i}=\varnothing$. The boundaries $\Gamma_{e}$ and $\Gamma_{i}$ are non-empty and of positive measure. Let us consider in $\Omega$ the state $z$ and the control $\left(\mathrm{v}_{1} ; \mathrm{v} 2\right)$

linked by the relations:

$$
\left\{\begin{array}{c}
\Delta z=0 \text { in } \Omega \\
z=v_{1}, \frac{\partial z}{\partial v}=v_{2} \text { on } \Gamma_{e}
\end{array}\right.
$$

With $z \in L^{2}(\Omega)$ and $\left(\mathrm{v}_{1} ; \mathrm{v}_{2}\right) \in L^{2}\left(\Gamma_{e}\right) \times L^{2}\left(\Gamma_{e}\right)$.

Problem (1) is a Cauchy problem for an elliptic operator (here the Laplacian operator). In general, problem (1) does not admit a solution and there is the instability of the solution when it exists, see for instance [6], [11]. It is so an ill-posed problem. But, it is important to control the Cauchy data, considering the fact that, such problems come from many concrete situations. It is the case in gravimetry for instance.
In the evolution case we have enzymatic reactions, see [4], and the bibliography of this work, the control of the transmission of electrical energy, the control of the form of plasmas. Let $U_{a d}^{1}$ and $U_{a d}^{2}$ be two no empty closed convex subsets of $L^{2}\left(\Gamma_{e}\right)$. We denote by

$$
\begin{array}{r}
A=\left\{\left(\left(v_{1}, v_{2}\right) ; z\right) \in U_{a d}^{1} \times U_{a d}^{2} \times L^{2}(\Omega), \Delta z\right. \\
\left.=0 \text { in } \Omega,\left.z\right|_{\Gamma_{e}}=v_{1},\left.\frac{\partial z}{\partial v}\right|_{\Gamma_{e}}=v_{2}\right\}
\end{array}
$$

a subset of $L^{2}\left(\Gamma_{e}\right) \times L^{2}\left(\Gamma_{e}\right) \times L^{2}(\Omega)$.

We assume that

$$
A \neq \varnothing
$$

It is obvious that $A$ is a closed convex subset of $L^{2}\left(\Gamma_{e}\right) \times$ $L^{2}\left(\Gamma_{e}\right) \times L^{2}(\Omega)$.

A couple of control-state $\left(\left(v_{1}, v_{2}\right) ; z\right)$ will be called "admissible couple" if it belongs in $A$. To simplify the notation, we will write $\left(v_{1}, v_{2} ; z\right)$ instead of $\left(\left(v_{1}, v_{2}\right) ; z\right)$. 
$\left\{\begin{array}{c}U_{a d}^{1}=L^{2}\left(\Gamma_{e}\right) \\ U_{a d}^{2}=\text { no empty closed convex subset of } L^{2}\left(\Gamma_{e}\right) .\end{array}\right.$

Then $A \neq \emptyset$ if $\left.z\right|_{\Gamma_{i}} \in L^{2}\left(\Gamma_{i}\right)$, we build $\varsigma$ solution of

$$
\left\{\begin{array}{c}
\Delta \varsigma=0 \text { in } \Omega \\
\varsigma=0 \text { on } \Gamma_{e} ; \frac{\partial \zeta}{\partial v}=v_{1} \text { on } \Gamma_{e}
\end{array}\right.
$$

The system (4) defines a unique $\varsigma \in H^{\frac{3}{2}}(\Omega)$. Therefore $\varsigma$

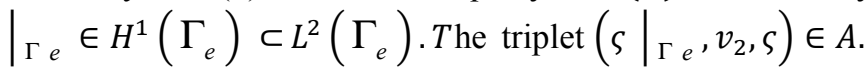
See [6] p. 416. Let us consider now, a strictly convex cost functional $\mathrm{J}$, defined for all admissible couple control-state $\left(v_{1}, v_{2} ; z\right)$ by

$$
J\left(v_{1}, v_{2}, z\right)=\frac{1}{2}\left\|z-z_{d}\right\|_{L^{2}(\Omega)}^{2}+\frac{N_{1}}{2}\left\|v_{1}\right\|_{L^{2}(\Omega)}^{2}+\frac{N_{2}}{2}\left\|v_{1}\right\|_{L^{2}(\Omega)}^{2}
$$

Where $\left(\mathrm{N}_{1}, \mathrm{~N}_{2}\right) \in \mathbb{R}_{+}^{*} \mathrm{x} \mathbb{R}_{+}^{*}$ and $z_{d} \in L^{2}(\Omega)$ the desired state.

We are then interested in the problem:

$$
\inf J\left(v_{1}, v_{2} ; z\right),\left(v_{1}, v_{2} ; z\right) \in A .
$$

problem (6) has a unique solution $\left(u_{1}, u_{2} ; y\right)$ which we are going to characterize. The function $J$ being differentiable, if $\left(u_{1}, u_{2} ; y\right)$ is the optimal control-state the first order EulerLagrange conditions gives.

The assumption (2) and the structure of J, show easily that

$$
\left\{\begin{array}{c}
\text { for all }\left(v_{1}, v_{2} ; z\right) \in A, \\
\int_{\Omega}\left(y-z_{d}\right)(z-y) d x+\int_{\Gamma_{e}} N_{1} u_{1}\left(v_{1}-u_{1}\right) d \Gamma_{e}+\int_{\Gamma_{e}} N_{2} u_{2}\left(v_{2}-u_{2}\right) d \Gamma_{e}
\end{array}\right.
$$

Many authors have already studied Cauchy systems.

In the parabolic and hyperbolic case we can cite M. Barry and O. Nakoulima in [1]; J. P. Kernevez [4]; M. Barry, O. Nakoulima and G. B. Ndiaye [2].

In the elliptic case we can cite J. L. Lions in [6]; O. Nakoulima in [9]; G.

Mophou and O. Nakoulima in [10].

To get a system where state and control are independent, J. L. Lions proposed in [5] a method of approximation by penalization. He obtained the convergence of the process when $U_{a d}^{1}=\mathrm{L}^{2}\left(\Gamma_{e}\right)$ and $U_{a d}^{2}=\mathrm{L}^{2}\left(\Gamma_{e}\right)$.

O. Nakoulima in [9] uses the regularization-penalization method. That means, he considered the control problem as a "singular" limit of a sequence of well-posed control problems. The convergence of the process is also obtained by the author in a particular case: $U_{a d}^{1}=U_{a d}^{2}=\left(\mathrm{L}^{2}\left(\Gamma_{e}\right)\right)^{+}$.

O. Nakoulima and G. Mophou in [10] use a regularization method that consists of viewing a singular problem as a limit of a family of well-posed problems. Following this method and assuming that the interior of considered convex is -nonempty, they obtain a singular optimality system (S. O. S.) for the considered control problem.

In this paper, we propose to give an approximate solution without an additional condition on the set of admissible couple-state.

We associate with the control problem of the ill-posed problem (1) -(2), (5) - (7) a family of hierarchical controls of well-posed problems.

The rest of this paper is organized as follows. In section 2.1 and section 2.2 , we establish respectively the optimality conditions of the follower and of the leader. In section 2.3, we study the equivalence of the problem studied in sections 2.1 and 2.2, and the problem (1), (5) - (7).

\section{Stackelberg Control of the Cauchy Problem for a Laplacian Operator}

In this part, we study two well-posed systems associated with the ill-posed problem (1). We propose as an alternative the Stackelberg control.

The Stackelberg leadership model is a multiple-objective optimization approach proposed by H. Von Stackelberg in [12]. This model involves two companies (controls) that compete on the market of the same product. The first (leader) to act must integrate the reaction of the other firm (followers) in the choices it makes in the amount of product that decides to put on the market. In this case, the control of Stackelberg consists of the hierarchical control of two objective functions, one by the follower and the other by the leader.

More precisely, we consider the following systems:

$$
\begin{gathered}
\left\{\begin{array}{c}
\Delta z_{1}=0 \text { in } \Omega \\
z_{1}=v_{1} \text { on } \Gamma_{e} ; \frac{\partial z_{1}}{\partial v}=\omega_{1} \text { on } \Gamma_{i}
\end{array}\right. \\
\left\{\begin{array}{c}
\Delta z_{2}=0 \text { in } \Omega \\
\frac{\partial z_{2}}{\partial v}=v_{2} \text { on } \Gamma_{e} ; z_{2}=\omega_{2} \text { on } \Gamma_{i}
\end{array}\right.
\end{gathered}
$$

Where $\left(z_{1}, z_{2}\right) \in L^{2}(\Omega) \times L^{2}(\Omega),\left(\omega_{1}, \omega_{2}\right) \in \mathrm{L}^{2}\left(\Gamma_{i}\right) \times$ $\mathrm{L}^{2}\left(\Gamma_{i}\right)$ with $\left(v_{1}, v_{2}\right)$ defined as in $(7)$.

Remark 2 Problems (8) and (9) are well-posed.

$$
\text { If }\left(\left.z_{1}\right|_{\Gamma e} ;\left.z_{2}\right|_{\Gamma_{i}}\right)=\left(v ; \omega_{2}\right) \in L^{2}\left(\Gamma_{e}\right) \times L^{2}\left(\Gamma_{i}\right)
$$


and [5], [7] $\left(z_{1}, z_{2}\right) \in H^{\frac{1}{2}}(\Omega) \times H^{\frac{1}{2}}(\Omega) .(8)$ and (9) admit

$$
\left(\left.\frac{\partial z_{1}}{\partial v}\right|_{\Gamma_{i}} ;\left.\frac{\partial z_{2}}{\partial v}\right|_{\Gamma_{e}}\right)=\left(\omega_{1}, v_{2}\right) \in L^{2}\left(\Gamma_{e}\right) \times L^{2}\left(\Gamma_{i}\right)
$$

some unique solutions $z_{1}$ and $z_{2}$ in $H^{\frac{1}{2}}(\Omega)$.

We consider now the cost function define by

for the trace theorem, see

$$
J_{2}\left(v_{1}, v_{2}, w_{1}, w_{2}, z_{1}\left(w_{1}\right), z_{2}\left(w_{2}\right)\right)=\frac{1}{2}\left\|\frac{\partial z_{1}}{\partial v}\left(w_{1}\right)-\frac{\partial z_{2}}{\partial v}\left(w_{2}\right)\right\|_{L^{2}\left(\Gamma_{i}\right)}^{2}+\frac{1}{2}\left\|z_{1}-z_{2}\right\|_{L^{2}\left(\Gamma_{e}\right)}^{2}
$$

Where $\left(z_{1}, z_{2}\right) \in L^{2}(\Omega) \times L^{2}(\Omega)$ is a solution of (8)-(9). And the minimization follow problem

$$
\min J_{2}\left(v_{1}, v_{2}, w_{1}, w_{2}, z_{1}\left(w_{1}\right), z_{2}\left(w_{2}\right)\right) ;\left(\omega_{1}, \omega_{2}\right) \in \mathrm{L}^{2}\left(\Gamma_{i}\right) \times \mathrm{L}^{2}\left(\Gamma_{i}\right)
$$

Remark 3 In a symmetrical way it is possible to introduce the functional cost

$$
J_{2}\left(v_{1}, v_{2}, w_{1}, w_{2}, z_{1}\left(w_{1}\right), z_{2}\left(w_{2}\right)\right)=\frac{1}{2}\left\|\frac{\partial z_{1}}{\partial v}\left(w_{1}\right)-\frac{\partial z_{2}}{\partial v}\left(w_{2}\right)\right\|_{L^{2}\left(\Gamma_{e}\right)}^{2}+\frac{1}{2}\left\|z_{1}-z_{2}\right\|_{L^{2}\left(\Gamma_{i}\right)}^{2}
$$

The set $A$ being non-empty, we now show that the resolution of the problem

(1) is equivalent to that of the problem (8) - (11).

Proposition 4 Assume that the problem (1) admits a solution. Then, solving problem (1) is equivalent to solve the problem (8) - (11) and

$$
\min J_{2}\left(v_{1}, v_{2}, \omega_{1}, \omega_{2}, z_{1}\left(\omega_{1}\right), z_{2}\left(\omega_{2}\right)\right)=0,\left(\omega_{1}, \omega_{2}\right) \in \mathrm{L}^{2}\left(\Gamma_{i}\right) \times \mathrm{L}^{2}\left(\Gamma_{i}\right)
$$

Proof Let $\left(v_{1}, v_{2}, z\right)$ be a solution of (1), such that $w_{1}=\frac{\partial z}{\partial v}$ on $\Gamma_{i}$ and $w_{2}=z$ on $\Gamma_{i}$ Then, we have

$$
J_{2}\left(v_{1}, v_{2}, w_{1}, w_{2}, z, z\right)=\frac{1}{2}\left\|w_{1}-\frac{\partial z}{\partial v}\right\|_{L^{2}\left(\Gamma_{i}\right)}^{2}+\frac{1}{2}\left\|v_{1}-z\right\|_{L^{2}\left(\Gamma_{e}\right)}^{2}=0 .
$$

Conversely, let $\left(v_{1}, v_{2}, w_{1}^{*}, w_{2}^{*}, z_{1}^{*}, z_{2}^{*}\right)$ be a solution of (8)-(11), we have

$$
0 \leq J_{2}\left(v_{1}, v_{2}, w_{1}^{*}, w_{2}^{*}, z_{1}^{*}, z_{2}^{*}\right) \leq J_{1}\left(v_{1}, v_{2}, w_{1}, w_{2}, z, z\right)=0
$$

(14)implies that $z_{1}^{*}=z_{2}^{*}$ on $\Gamma_{e}$ and $\frac{\partial z_{1}^{*}}{\partial v}=\frac{\partial z_{2}^{*}}{\partial v}$ on $\Gamma_{i}$. We deduce that,

$z_{1}^{*}-z_{2}^{*}$ is the solution of the following problem

$$
\left\{\begin{array}{c}
\Delta\left(z_{1}^{*}-z_{2}^{*}\right)=0 \text { in } \Omega \\
z_{1}^{*}-z_{2}^{*}=0 \text { on } \Gamma_{e} ; \frac{\partial z_{1}^{*}}{\partial v}-\frac{\partial z_{2}^{*}}{\partial v}=0 \text { on } \Gamma_{i}
\end{array}\right.
$$

from (15), we obtain $z_{1}^{*}=z_{2}^{*}=z$ in $\Omega$.

\subsection{Optimality System of the Follower}

Proposition 5 The functional $J_{2}$ is twice Gâteaux differentiable and strictly

$$
\text { convex. }
$$

Proof. We first show that $J_{2}$ is Gâteaux differentiable.

Let $\left(\varphi_{1}, \varphi_{2}\right) \in L^{2}\left(\Gamma_{i}\right) \times L^{2}\left(\Gamma_{i}\right), \lambda$

$\in \mathbb{R}$ and denote by $(.,$.$) the scalar$

product in $L^{2}$.

$$
\begin{gathered}
J_{2}\left(v_{1}, v_{2}, \omega_{1}+\lambda \varphi_{1}, \omega_{2}+\lambda \varphi_{2}, z_{1}, z_{2}\right) \\
=\frac{1}{2}\left\|\frac{\partial z_{1}}{\partial v}\left(\omega_{1}+\lambda \varphi_{1}\right)-\frac{\partial z_{2}}{\partial v}\left(\omega_{2}+\lambda \varphi_{2}\right)\right\|_{L^{2}\left(\Gamma_{i}\right)}^{2}+\frac{1}{2}\left\|v_{1}-z_{2}\left(\omega_{2}+\lambda \varphi_{2}\right)\right\|_{L^{2}\left(\Gamma_{e}\right)}^{2}
\end{gathered}
$$

Let us consider the applications

$$
\begin{gathered}
\varphi_{1} \rightarrow z_{1}\left(\varphi_{1}\right)-z_{1}(0) \text { and } \varphi_{2} \rightarrow z_{2}\left(\varphi_{2}\right)-z_{2}(0) \\
\text { solution of } \\
\left\{\begin{array}{c}
\Delta \Phi_{1}=0 \text { in } \Omega \\
\Phi_{1}=0 \text { on } \Gamma_{e} ; \frac{\partial \Phi_{1}}{\partial v}=\Phi_{1} \text { on } \Gamma_{i}
\end{array}\right. \\
\left\{\begin{array}{c}
\Delta \Phi_{2}=0 \text { in } \Omega \\
\frac{\partial \Phi_{2}}{\partial v}=0 \text { on } \Gamma_{e} ; \Phi_{2}=\varphi_{2} \text { on } \Gamma_{i}
\end{array}\right.
\end{gathered}
$$

The applications defined in (16) are linear. The following calculations are performed 


$$
\begin{gathered}
=\frac{1}{2}\left\|\omega_{1}-\frac{\partial z_{2}}{\partial v}\left(w_{2}\right)\right\|_{L^{2}\left(\Gamma_{i}\right)}^{2}+\frac{\lambda^{2}}{2}\left\|\varphi_{1}-\frac{\partial z_{2}}{\partial v}\left(\varphi_{2}\right)+\frac{\partial z_{2}}{\partial v}(0)\right\|_{L^{2}\left(\Gamma_{i}\right)}^{2}+\frac{1}{2}\left\|v_{1}-z_{2}\left(\omega_{2}\right)\right\|_{L^{2}\left(\Gamma_{e}\right)}^{2}+ \\
\frac{\lambda^{2}}{2}\left\|-z_{2}\left(\varphi_{2}\right)+z_{2}(0)\right\|_{L^{2}\left(\Gamma_{e}\right)}^{2}+\lambda\left(\omega_{1}-\frac{\partial z_{2}}{\partial v}\left(\omega_{2}\right), \varphi_{1}-\frac{\partial z_{2}}{\partial v}\left(\varphi_{2}\right)+\frac{\partial z_{2}}{\partial v}(0)\right)_{L^{2}\left(\Gamma_{i}\right)} \\
+\lambda\left(v_{1}-z_{2}\left(\omega_{2}\right),-z_{2}\left(\varphi_{2}\right)+z_{2}(0)\right)_{L^{2}\left(\Gamma_{e}\right)}
\end{gathered}
$$

Hence

$$
\begin{gathered}
\lim _{\lambda \rightarrow 0} \frac{J_{2}\left(\omega_{1}+\lambda \varphi_{1}, \varphi_{2}+\lambda \varphi_{2}, v_{1}, v_{2}, z_{1}, z_{2}\right)-J_{2}\left(\omega_{1}, \varphi_{2}, v_{1}, v_{2}, z_{1}, z_{2}\right)}{\lambda} \\
=\left(\omega_{1}-\frac{\partial z_{2}}{\partial v}\left(\omega_{2}\right), \varphi_{1}-\frac{\partial z_{2}}{\partial v}\left(\varphi_{2}\right)+\frac{\partial z_{2}}{\partial v}(0)\right)_{L^{2}\left(\Gamma_{i}\right)}+\left(v_{1}-z_{2}\left(\omega_{2}\right),-z_{2}\left(\varphi_{2}\right)+z_{2}(0)\right)_{L^{2}\left(\Gamma_{e}\right)}
\end{gathered}
$$

This last result shows that $J_{2}$ is Gâteaux differentiable and

$$
\begin{aligned}
d J_{2}\left(v_{1}, v_{2}, \omega_{1}, \omega_{2}, z_{1}, z_{2}\right) \cdot\left(\varphi_{1}, \varphi_{2}\right)= & \left(\omega_{1}-\frac{\partial z_{2}}{\partial v}\left(\omega_{2}\right), \varphi_{1}-\frac{\partial z_{2}}{\partial v}\left(\varphi_{2}\right)+\frac{\partial z_{2}}{\partial v}(0)\right)_{L^{2}\left(\Gamma_{i}\right)}+ \\
& \left(v_{1}-z_{2}\left(\omega_{2}\right),-z_{2}\left(\varphi_{2}\right)+z_{2}(0)\right)_{L^{2}\left(\Gamma_{e}\right)}
\end{aligned}
$$

For $\left(\xi_{1}, \xi_{2}\right) \in L^{2}\left(\Gamma_{i}\right) \times L^{2}\left(\Gamma_{i}\right)$ and $\lambda \in \mathbb{R}$ we have

$$
\begin{gathered}
d J_{2}\left(v_{1}, v_{2}, \omega_{1}+\lambda \xi_{1}, \omega_{2}+\lambda \xi_{2}, z_{1}, z_{2}\right) \cdot\left(\varphi_{1}, \varphi_{2}\right) \\
=\left(\omega_{1}+\lambda \xi_{1}-\frac{\partial z_{2}}{\partial v}\left(\omega_{2}+\lambda \xi_{2}\right), \varphi_{1}-\frac{\partial z_{2}}{\partial v}\left(\varphi_{2}\right)+\frac{\partial z_{2}}{\partial v}(0)\right)_{L^{2}\left(\Gamma_{i}\right)} \\
+\left(v_{1}-z_{2}\left(\omega_{2}+\lambda \xi_{2}\right),-z_{2}\left(\varphi_{2}\right)+z_{2}(0)\right)_{L^{2}\left(\Gamma_{e}\right)} \\
=\left(\omega_{1}-\frac{\partial z_{2}}{\partial v}\left(\omega_{2}\right), \varphi_{1}-\frac{\partial z_{2}}{\partial v}\left(\varphi_{2}\right)+\frac{\partial z_{2}}{\partial v}(0)\right)_{L^{2}\left(\Gamma_{i}\right)} \\
+\left(\lambda \xi_{1}-\frac{\partial z_{2}}{\partial v}\left(\xi_{2}\right)+\frac{\partial z_{2}}{\partial v}(0), \varphi_{1}-\frac{\partial z_{2}}{\partial v}\left(\varphi_{2}\right)+\frac{\partial z_{2}}{\partial v}(0)\right)_{L^{2}\left(\Gamma_{i}\right)} \\
+\left(v_{1}-z_{2}\left(\omega_{2}\right),-z_{2}\left(\varphi_{2}\right)+z_{2}(0)\right)_{L^{2}\left(\Gamma_{e}\right)} \\
+\lambda\left(-z_{2}\left(\xi_{2}\right)+z_{2}(0),-z_{2}\left(\omega_{2}\right)+z_{2}(0)\right)_{L^{2}\left(\Gamma_{e}\right)}
\end{gathered}
$$

Which give

$$
\begin{gathered}
\lim _{\lambda \rightarrow 0}\left(\frac{J_{2}\left(v_{1}, v_{2}, \omega_{1}+\lambda \xi_{1}, \omega_{2}+\lambda \xi_{2}, z_{1}, z_{2}\right) \cdot\left(\varphi_{1}, \varphi_{2}\right)}{\lambda}-\frac{J_{2}\left(\omega_{1}, \omega_{2}, v_{1}, v_{2}, z_{1}, z_{2}\right) \cdot\left(\varphi_{1}, \varphi_{2}\right)}{\lambda}\right) \\
=\left(\frac{\partial z_{1}}{\partial v}\left(\xi_{1}\right)-\frac{\partial z_{1}}{\partial v}(0)-\frac{\partial z_{2}}{\partial v}\left(\xi_{2}\right)+\frac{\partial z_{2}}{\partial v}(0), \frac{\partial z_{1}}{\partial v}\left(\varphi_{1}\right)-\frac{\partial z_{1}}{\partial v}(0)-\frac{\partial z_{2}}{\partial v}\left(\varphi_{2}\right)+\frac{\partial z_{2}}{\partial v}(0)\right)_{L^{2}\left(\Gamma_{i}\right)} \\
+\left(-z_{2}\left(\xi_{2}\right)+z_{2}(0),-z_{2}\left(\omega_{2}\right)+z_{2}(0)\right)_{L^{2}\left(\Gamma_{e}\right)}
\end{gathered}
$$

and so

$$
\begin{gathered}
d J_{2}^{2}\left(v_{1}, v_{2}, \omega_{1}, \omega_{2}, z_{1}, z_{2}\right) \cdot\left(\xi_{1}, \xi_{2}\right) \\
=\left(\xi_{1}-\frac{\partial z_{2}}{\partial v}\left(\xi_{2}\right)+\frac{\partial z_{2}}{\partial v}(0), \varphi_{1}-\frac{\partial z_{2}}{\partial v}\left(\varphi_{2}\right)+\frac{\partial z_{2}}{\partial v}(0)\right)_{L^{2}\left(\Gamma_{i}\right)} \\
+\left(-z_{2}\left(\xi_{2}\right)+z_{2}(0),-z_{2}\left(\omega_{2}\right)+z_{2}(0)\right)_{L^{2}\left(\Gamma_{e}\right) .}
\end{gathered}
$$

This last result shows that $J_{2}$ is twice Gâteaux differentiable.

$$
\begin{gathered}
=\left\|\varphi_{1}-\frac{\partial z_{2}}{\partial v}\left(\varphi_{2}\right)+\frac{\partial z_{2}}{\partial v}(0)\right\|_{L^{2}\left(\Gamma_{i}\right)}^{2} \\
+\left\|-z_{2}\left(\varphi_{2}\right)+z_{2}(0)\right\|_{L^{2}\left(\Gamma_{i}\right)}^{2}
\end{gathered}
$$


As $d J_{2}^{2}\left(v_{1}, v_{2}, \omega_{1}, \omega_{2}, z_{1}, z_{2}\right) \cdot\left(\varphi_{1}, \varphi_{2}\right) \cdot\left(\varphi_{1}, \varphi_{2}\right) \geq 0$, then $J_{2}$ is convex.

In addition, we have $d J_{2}^{2}\left(v_{1}, v_{2}, \omega_{1}, \omega_{2}, z_{1}, z_{2}\right) \cdot\left(\varphi_{1}, \varphi_{2}\right) \cdot$ $\left(\varphi_{1}, \varphi_{2}\right)=0$

Implies that $\varphi_{1}-\frac{\partial z_{2}}{\partial v}\left(\varphi_{2}\right)+\frac{\partial z_{2}}{\partial v}(0)=0$ on $\Gamma_{i}$ and $z_{2}\left(\varphi_{2}\right)-$ $z_{2}(0)=0$ on $\Gamma_{e}$.

From (18) and the identity $z_{2}\left(\varphi_{2}\right)-z_{2}(0)=0$ on $\Gamma_{e}$, the uniqueness of the Cauchy problem gives

$$
z_{2}\left(\varphi_{2}\right)-z_{2}(0)=0 \text { in } \bar{\Omega} \text { and thus } \varphi_{2}=0 . \mathrm{F}
$$

From the identity

$$
\varphi_{1}-\frac{\partial z_{2}}{\partial v}\left(\varphi_{2}\right)+\frac{\partial z_{2}}{\partial v}(0)=0 \text { on } \Gamma_{i} \text { we have } \varphi_{1}=0
$$

Finally $J_{2}$ is strictly convex.

The strict convexity of $J_{2}$ leads to the uniqueness of the solution of prob-lem (11).

We introduce now some adjoint systems of (8) and (9) define by

$$
\begin{gathered}
\left\{\begin{array}{c}
\Delta p_{1}=0 \text { in } \Omega \\
p_{1}=v_{1}-z_{2} \text { on } \Gamma_{e} ; \frac{\partial p_{1}}{\partial v}=-\omega_{1}+\frac{\partial p_{2}}{\partial v} \text { on } \Gamma_{i}
\end{array}\right. \\
\Delta p_{2}=0 \text { in } \Omega \\
\left\{\begin{array}{c}
\frac{\partial p_{2}}{\partial v}=v_{1}-z_{2} \text { on } \Gamma_{e} ; p_{2}=-\omega_{1}+\frac{\partial p_{2}}{\partial v} \text { on } \Gamma_{i}
\end{array}\right.
\end{gathered}
$$

We obtain the optimality system below

Proposition $6\left(\omega_{1}^{*}, \omega_{2}^{*}\right)$ is an optimal solution of (13) if and only if it exists

$\left(z_{1}^{*}, z_{2}^{*}\right)$ satisfying (8)- (9) and $\left(p_{1}^{*}, p_{2}^{*}\right)$ satisfying (20)-(21) such as the

triplet $\left\{\left(\omega_{1}^{*}, \omega_{2}^{*}\right),\left(z_{1}^{*}, z_{2}^{*}\right),\left(p_{1}^{*}, p_{2}^{*}\right)\right\}$ is the solution of the optimality systems:

$$
\left\{\begin{array}{c}
\Delta p_{1}^{*}=0 \text { in } \Omega \\
z_{1}^{*}=v_{1} \text { on } \Gamma_{e} ; \frac{\partial z_{1}^{*}}{\partial v}=-\omega_{1}^{*} \text { on } \Gamma_{i}
\end{array}\right.
$$

$$
\begin{aligned}
& \left\{\begin{array}{c}
\Delta p_{2}=0 \text { in } \Omega \\
\frac{\partial z_{2}}{\partial v}=v_{2} \text { on } \Gamma_{e} ; z_{2}^{*}=\omega_{2}^{*} \text { on } \Gamma_{i}
\end{array}\right. \\
& \left\{\begin{array}{c}
\Delta p_{1}^{*}=0 \text { in } \Omega \\
p_{1}^{*}=v_{1}-z_{2}^{*} \text { on } \Gamma_{e} ; \frac{\partial p_{1}^{*}}{\partial v}=-\omega_{1}^{*}+\frac{\partial z_{2}^{*}}{\partial v} \text { on } \Gamma_{i}
\end{array}\right. \\
& \left\{\begin{array}{c}
\Delta p_{2}^{*}=0 \text { in } \Omega \\
\frac{\partial p_{2}^{*}}{\partial v}=v_{1}-z_{2}^{*} \text { on } \Gamma_{e} ; p_{2}^{*}=-\omega_{1}^{*}+\frac{\partial z_{2}^{*}}{\partial v} \text { on } \Gamma_{i}
\end{array}\right.
\end{aligned}
$$

and

$$
\frac{\partial p_{1}^{*}}{\partial v}=0 \text { on } \Gamma_{i} ; \frac{\partial p_{2}^{*}}{\partial v}=0 \text { on } \Gamma_{i}
$$

Proof.Let $\left(\omega_{1}, \omega_{2},\right),\left(\varphi_{1}, \varphi_{2}\right) \in L^{2}\left(\Gamma_{i}\right) \times L^{2}\left(\Gamma_{i}\right), \lambda \in \mathbb{R}$. From (19) we have

$$
\begin{gathered}
d J_{2}\left(v_{1}, v_{2}, \omega_{1}, \omega_{2}, z_{1}, z_{2}\right) \cdot\left(\varphi_{1}, \varphi_{2}\right) \\
=\left(\omega_{1}-\frac{\partial z_{2}}{\partial v}\left(\omega_{2}\right), \varphi_{1}-\frac{\partial z_{2}}{\partial v}\left(\varphi_{2}\right)+\frac{\partial z_{2}}{\partial v}(0)\right)_{L^{2}\left(\Gamma_{i}\right)}+\left(v_{1}-\right. \\
\left.z_{2}\left(\omega_{2}\right),-z_{2}\left(\varphi_{2}\right)+z_{2}(0)\right)_{L^{2}\left(\Gamma_{e}\right)}
\end{gathered}
$$

According to the Euler optimality conditions, $\left(\omega_{1}^{*}, \omega_{2}^{*}\right)$ is the optimal solution of (13) if and only if,

$$
\begin{aligned}
& \left(\varphi_{1}, \varphi_{2}\right) \in L^{2}\left(\Gamma_{i}\right) \times L^{2}\left(\Gamma_{i}\right), d J_{2}\left(v_{1}, v_{2}, \omega_{1}^{*}, \omega_{2}^{*}, z_{1}^{*}, z_{2}^{*}\right) \\
& \cdot\left(\varphi_{1}, \varphi_{2}\right) \geq 0 \text {. } \\
& \text { that is, } \\
& \forall\left(\varphi_{1}, \varphi_{2}\right) \in L^{2}\left(\Gamma_{i}\right) \times L^{2}\left(\Gamma_{i}\right),\left(\omega_{1}^{*}-\frac{\partial z_{2}^{*}}{\partial v}, \varphi_{1}-\frac{\partial z_{2}}{\partial v}\left(\varphi_{2}\right)\right. \\
& \left.+\frac{\partial z_{2}}{\partial v}(0)\right)_{L^{2}\left(\Gamma_{i}\right)} \\
& +\left(v_{1}-z_{2}^{*},-z_{2}\left(\varphi_{2}\right)+z_{2}(0)\right)_{L^{2}\left(\Gamma_{e}\right)} \geq 0 \text {. }
\end{aligned}
$$

Hence

$$
\begin{gathered}
\left(\omega_{1}^{*}-\frac{\partial z_{2}^{*}}{\partial v}, \varphi_{1}\right)_{L^{2}\left(\Gamma_{i}\right)}+\left(\omega_{1}^{*}-\frac{\partial z_{2}^{*}}{\partial v}-\frac{\partial z_{2}}{\partial v}\left(\varphi_{2}\right)+\frac{\partial z_{2}}{\partial v}(0)\right)_{L^{2}\left(\Gamma_{i}\right)} \\
+\left(v_{1}-z_{2}^{*},-z_{2}\left(\varphi_{2}\right)+z_{2}(0)\right)_{L^{2}\left(\Gamma_{e}\right)} \geq 0
\end{gathered}
$$

As a result, talking into account (24) and (25) we have

$$
\begin{gathered}
\left(-\frac{\partial p_{1}^{*}}{\partial v}, \varphi_{1}\right)_{L^{2}\left(\Gamma_{i}\right)}+\left(p_{2}^{*}, \frac{\partial z_{2}}{\partial v}\left(\varphi_{2}\right)-\frac{\partial z_{2}}{\partial v}(0)\right)_{L^{2}\left(\Gamma_{i}\right)} \\
--\left(\frac{\partial p_{2}^{*}}{\partial v}, z_{2}\left(\varphi_{2}\right)-z_{2}(0)\right)_{L^{2}\left(\Gamma_{e}\right)} \geq 0 .
\end{gathered}
$$

(18)

And integrating by parts we have

$-\int_{\Gamma_{e}} \frac{\partial p_{2}^{*}}{\partial v} \Phi_{2} d s+\int_{\Gamma_{i}} p_{2}^{*} \frac{\partial \Phi_{2}}{\partial v} d s=\int_{\Gamma_{i}} \frac{\partial p_{2}^{*}}{\partial v} \Phi_{2} d s$.

$$
\begin{aligned}
\forall\left(\varphi_{1}, \varphi_{2}\right) & \in \mathrm{L}^{2}\left(\Gamma_{i}\right) \times \mathrm{L}^{2}\left(\Gamma_{i}\right),\left(-\frac{\partial p_{1}^{*}}{\partial v}, \varphi_{1}\right)_{L^{2}\left(\Gamma_{i}\right)} \\
& +\left(\frac{\partial p_{2}^{*}}{\partial v}, \varphi_{2}\right)_{L^{2}\left(\Gamma_{i}\right)}=0 .
\end{aligned}
$$

(26) become 
Finally we have $\frac{\partial p_{1}^{*}}{\partial v}=0$ on $\Gamma_{i}$ and $\frac{\partial p_{2}^{*}}{\partial v}=0$ on $\Gamma_{i}$.

\subsection{Optimality System of the Leader}

Consider the cost function

$$
J_{1}\left(v_{1}, v_{2}, \omega_{1}^{*}, \omega_{2}^{*}, z_{1}^{*}, z_{2}^{*}\right)=\frac{\theta_{1}}{2}\left\|z_{1}^{*}-z_{d}\right\|_{L^{2}(\Omega)}^{2}+\frac{\theta_{2}}{2}\left\|z_{2}^{*}-z_{d}\right\|_{L^{2}(\Omega)}^{2}+\frac{N_{1}}{2}\left\|v_{1}\right\|_{L^{2}\left(\Gamma_{e}\right)}^{2}+\frac{N_{2}}{2}\left\|v_{2}\right\|_{L^{2}\left(\Gamma_{e}\right)}^{2}
$$

where $\left(N_{1}, N_{2}\right)$ and $z_{d}$ are defined in (5), $\theta_{1}$ and $\theta_{2}$ are some reals such

that $\theta_{1}+\theta_{2}=1$,

and the problem

$$
\begin{gathered}
\inf J_{1}\left(v_{1}, v_{2}, \omega_{1}^{*}, \omega_{2}^{*}, z_{1}^{*}, z_{2}^{*}\right),\left(v_{1}, v_{2}\right) \in L^{2}\left(\Gamma_{e}\right) \times L^{2}\left(\Gamma_{e}\right) \\
\text { Proposition } 7 \text { There existe }\left(u_{1}^{*}, u_{2}^{*}\right) \\
\in L^{2}\left(\Gamma_{e}\right) \times L^{2}\left(\Gamma_{e}\right) \text { unique such that } \\
\forall\left(v_{1}, v_{2}\right) \in L^{2}\left(\Gamma_{e}\right) \times L^{2}\left(\Gamma_{e}\right),(.) \\
J_{1}\left(u_{1}^{*}, u_{2}^{*}, \omega_{1}^{*}, \omega_{2}^{*}, z_{1}^{*}, z_{2}^{*}\right) \leq J_{1}\left(v_{1}, v_{2}, \omega_{1}^{*}, \omega_{2}^{*}, z_{1}^{*}, z_{2}^{*}\right)
\end{gathered}
$$

Proof. $L^{2}\left(\Gamma_{e}\right) \times L^{2}\left(\Gamma_{e}\right)$ is closed convex.

$J_{1}$ is coercive and strictly convex, then the problem (29) holds true.

Consider again one adjoint problem of (8) and (9) define by

$$
\begin{aligned}
& \left\{\begin{array}{c}
\Delta p_{1}=z_{1}^{*}-z_{d} \text { in } \Omega \\
p_{1}=0 \text { on } \Gamma_{e} ; \frac{\partial p_{1}}{\partial v}=0 \text { on } \Gamma_{i}
\end{array}\right. \\
& \left\{\begin{array}{c}
\Delta p_{2}=z_{2}^{*}-z_{d} \text { in } \Omega \\
\frac{\partial p_{2}}{\partial v}=0 \text { on } \Gamma_{e} ; p_{2}=0 \text { on } \Gamma_{i}
\end{array}\right.
\end{aligned}
$$

We obtain the optimality system below

Proposition $8\left(u_{1}^{*}, u_{2}^{*}\right)$ is an optimal solution of (28) if and only if it exists

$\left(z_{1}^{*} ; z_{2}^{*}\right)$ satisfying $(8)-(9)$ and $\left(p_{1}^{*} ; p_{2}^{*}\right)$ satisfying $(30)-(31)$ such as the triplet $\left\{\left(u_{1}^{*} ; u_{2}^{*}\right),\left(z_{1}^{*} ; z_{2}^{*}\right),\left(p_{1}^{*} ; p_{2}^{*}\right)\right\}$ is the solution of the optimality systems:

$$
\begin{gathered}
\left\{\begin{array}{c}
\Delta z_{1}^{*}=0 \text { in } \Omega \\
z_{1}^{*}=u_{1}^{*} \text { on } \Gamma_{e} ; \frac{\partial z_{1}^{*}}{\partial v}=\omega_{1}^{*} \text { on } \Gamma_{i}
\end{array}\right. \\
\left\{\begin{array}{c}
\Delta z_{2}^{*}=0 \text { in } \Omega \\
\frac{\partial z_{2}^{*}}{\partial v}=u_{2}^{*} \text { on } \Gamma_{e} ; z_{2}^{*}=\omega_{2}^{*} \text { on } \Gamma_{i}
\end{array}\right. \\
\left\{\begin{array}{c}
\Delta p_{2}^{*}=z_{1}^{*}-z_{d} \text { in } \Omega \\
p_{1}^{*}=0 \text { on } \Gamma_{e} ; \frac{\partial p_{1}^{*}}{\partial v}=0 \text { on } \Gamma_{i}
\end{array}\right. \\
\left\{\begin{array}{c}
\Delta p_{2}^{*}=z_{2}^{*}-z_{d} \text { in } \Omega \\
\frac{\partial p_{2}}{\partial v}=0 \text { on } \Gamma_{e} ; p_{2}^{*}=0 \text { on } \Gamma_{i}
\end{array}\right.
\end{gathered}
$$

And

$$
-\theta_{1} \frac{\partial p_{1}^{*}}{\partial v}+N_{1} u_{1}^{*}=0 \text { on } \Gamma_{e} ; \theta_{2} p_{2}^{*}+N_{2} u_{2}^{*}=0 \text { on } \Gamma_{e}
$$

Proof. Let $\left(\varphi_{1}, \varphi_{2}\right) \in L^{2}\left(\Gamma_{e}\right) \times L^{2}\left(\Gamma_{e}\right), \lambda \in \mathbb{R}$ and denote by (...) the scalar

Product in $L^{2}$.

Let us consider the applications

$$
\varphi_{1} \rightarrow \mathrm{z}_{1}\left(\varphi_{1}\right)-\mathrm{z}_{1}(0) \text { and } \varphi_{2} \rightarrow \mathrm{z}_{2}\left(\varphi_{2}\right)-\mathrm{z}_{2}(0)
$$

solutions of

$$
\begin{gathered}
\left\{\begin{array}{c}
\Delta \Phi_{1}=0 \text { in } \Omega \\
\Phi_{1}=\varphi_{1} \text { on } \Gamma_{e} ; \frac{\partial \Phi_{1}}{\partial v}=0 \text { on } \Gamma_{i}
\end{array}\right. \\
\left\{\begin{array}{c}
\Delta \Phi_{2}=0 \text { in } \Omega \\
\frac{\partial \Phi_{2}}{\partial v}=\varphi_{2} \text { on } \Gamma_{e} ; \Phi_{2}=0 \text { on } \Gamma_{i}
\end{array}\right.
\end{gathered}
$$

The applications define in (37) are linear.

We have

$$
\begin{gathered}
\forall\left(\varphi_{1}, \varphi_{2,}\right) \in L^{2}\left(\Gamma_{e}\right) \times L^{2}\left(\Gamma_{e}\right), \\
d J_{1}\left(v_{1}, v_{2,}\right) \cdot\left(\varphi_{1}, \varphi_{2,}\right) \\
=\theta_{1}\left(z_{1}^{*}\left(v_{1}\right)-z_{d}, z_{1}^{*}\left(\varphi_{1}\right)-z_{1}^{*}(0)\right)_{L^{2}(\Omega)} \\
+\theta_{2}\left(z_{2}^{*}\left(v_{2}\right)-z_{d}, z_{2}^{*}\left(\varphi_{1}\right)-z_{2}^{*}(0)\right)_{L^{2}(\Omega)} \\
+N_{1}\left(v_{1}, \varphi_{2},\right)_{L^{2}\left(\Gamma_{e}\right)}+N_{2}\left(v_{2}, \varphi_{2},\right)_{L^{2}\left(\Gamma_{e}\right)}
\end{gathered}
$$

According to the Euler optimality conditions, $\left(u_{1}^{*} ; u_{2}^{*}\right)$ is an optimal solution

of (28) if and only if,

$\forall\left(\varphi_{1}, \varphi_{2,}\right) \in L^{2}\left(\Gamma_{e}\right) \times L^{2}\left(\Gamma_{e}\right), d J_{1}\left(u_{1}^{*} ; u_{2}^{*}\right) \cdot\left(\varphi_{1}, \varphi_{2,}\right) \geq 0$.

That gives

$$
\begin{gathered}
\forall\left(\varphi_{1}, \varphi_{2,}\right) \in L^{2}\left(\Gamma_{e}\right) \times L^{2}\left(\Gamma_{e}\right), \\
\theta_{1}\left(z_{1}^{*}\left(u_{1}^{*}\right)-z_{d}, z_{1}^{*}\left(\varphi_{1}\right)-z_{1}^{*}(0)\right)_{L^{2}(\Omega)} \\
+\theta_{2}\left(z_{2}^{*}\left(u_{2}^{*}\right)-z_{d}, z_{2}^{*}\left(\varphi_{1}\right)-z_{2}^{*}(0)\right)_{L^{2}(\Omega)} \\
+N_{1}\left(u_{1}^{*}, \varphi_{1},\right)_{L^{2}\left(\Gamma_{e}\right)}+N_{2}\left(u_{2}^{*}, \varphi_{2},\right)_{L^{2}\left(\Gamma_{e}\right)} \geq 0 .
\end{gathered}
$$

Multiplying the firsts equations of (30) and (31) respectively by $\Phi_{1}$ and $\Phi_{2}$ (solutions of (38) and (39) and integrating by parts we obtain

$$
\left(z_{1}^{*}\left(u_{1}^{*}\right)-z_{d}, z_{1}^{*}\left(\varphi_{1}\right)-z_{1}^{*}(0)\right)_{L^{2}(\Omega)}=\left(-\frac{\partial p_{1}^{*}}{\partial v}, \varphi_{1}\right)_{L^{2}\left(\Gamma_{e}\right)}
$$

And

$$
\left(z_{2}^{*}\left(u_{2}^{*}\right)-z_{d}, z_{2}^{*}\left(\varphi_{2}\right)-z_{2}^{*}(0)\right)_{L^{2}(\Omega)}=\left(p_{2}^{*}, \varphi_{2}\right)_{L^{2}\left(\Gamma_{e}\right)}
$$

From (40)-(42) we obtain

$$
\begin{gathered}
\forall\left(\varphi_{1}, \varphi_{2,}\right) \in L^{2}\left(\Gamma_{e}\right) \times L^{2}\left(\Gamma_{e}\right), \\
\left(-\theta_{1} \frac{\partial p_{1}^{*}}{\partial v}+N_{1} u_{1}^{*}, \varphi_{1}\right)_{L^{2}\left(\Gamma_{e}\right)}+\left(\theta_{2} p_{2}^{*}+N_{2} u_{2}^{*}, \varphi_{2}\right)_{L^{2}\left(\Gamma_{e}\right)}=0 .
\end{gathered}
$$


and finally, we have

$$
-\theta_{1} \frac{\partial p_{1}^{*}}{\partial v}+N_{1} u_{1}^{*}=0 \text { on } \Gamma_{e} \text { and } \theta_{2} p_{2}^{*}+N_{2} u_{2}^{*}=0 \text { on } \Gamma_{e}
$$

\subsection{Equivalence to the Problem (1); (5)-(7)}

In this section, we will show that the optimality system (32)-(36) makes it possible to calculate the solution of problem (1), (5) - (7).

Let $\left(u_{1}, u_{2}, \mathrm{y}\right)$ be the unique solution of problem (1), (5) (7) and $\left(v_{1}, v_{2}\right) \in L^{2}\left(\Gamma_{e}\right) \times L^{2}\left(\Gamma_{e}\right)$.

$\mathrm{J}_{2}$ being the cost function defined in (10) and $\left(u_{1}^{*}, u_{2}^{*}\right)$;

$$
\begin{gathered}
J_{1}\left(u_{1}^{*}, u_{2}^{*}, \omega_{1}^{*}, \omega_{2}^{*}, z_{1}^{*}, z_{2}^{*}\right) \\
=\frac{\theta_{1}}{2}\left\|z_{1}^{*}-z_{d}\right\|_{L^{2}(\Omega)}^{2}+\frac{\theta_{2}}{2}\left\|z_{2}^{*}-z_{d}\right\|_{L^{2}(\Omega)}^{2}+\frac{N_{1}}{2}\left\|u_{1}^{*}\right\|_{L^{2}\left(\Gamma_{e}\right)}^{2}+\frac{N_{2}}{2}\left\|u_{2}^{*}\right\|_{L^{2}\left(\Gamma_{e}\right)}^{2} \\
=\frac{\theta_{1}}{2}\left\|y-z_{d}\right\|_{L^{2}(\Omega)}^{2}+\frac{\theta_{2}}{2}\left\|y-z_{d}\right\|_{L^{2}(\Omega)}^{2}+\frac{N_{1}}{2}\left\|u_{1}\right\|_{L^{2}\left(T_{e}\right)}^{2}+\frac{N_{2}}{2}\left\|u_{2}\right\|_{L^{2}\left(\Gamma_{e}\right)}^{2} \\
=\left(\frac{\theta_{1}}{2}+\frac{\theta_{2}}{2}\right)\left\|y-z_{d}\right\|_{L^{2}(\Omega)}^{2}+\frac{N_{1}}{2}\left\|u_{1}\right\|_{L^{2}\left(\Gamma_{e}\right)}^{2}+\frac{N_{2}}{2}\left\|u_{2}\right\|_{L^{2}\left(\Gamma_{e}\right)}^{2} \\
=\left\|y-z_{d}\right\|_{L^{2}(\Omega)}^{2}+\frac{N_{1}}{2}\left\|u_{1}\right\|_{L^{2}\left(T_{e}\right)}^{2}+\frac{N_{2}}{2}\left\|u_{2}\right\|_{L^{2}\left(T_{e}\right)}^{2} \\
=J\left(u_{1}, u_{2,} y\right)
\end{gathered}
$$

And finally,

$$
\begin{aligned}
& \inf J\left(u_{1}, u_{2}, y\right) \inf J_{1}\left(v_{1}, v_{2}, \omega_{1}^{*}, \omega_{2}^{*}, z_{1}^{*}, z_{2}^{*}\right) \\
& \left(v_{1}, v_{2}, z\right) \in A\left(v_{1}, v_{2}\right) \in L^{2}\left(\Gamma_{e}\right) \times L^{2}\left(\Gamma_{e}\right) .
\end{aligned}
$$

\section{Conclusion}

The present article presents an alternative possibility to study the optimal control problem (6) and to obtain an optimality condition where state and control are independent, different from the regularization-penalization method introduced by $O$. Nakoulima in [9] or the regularization method due to O. Nakoulima and G. Mophou in [10].

\section{Acknowledgements}

The author wishes to express his gratitude to Professor Ousseynou

Nakoulima for his many helpful suggestions.

\section{References}

[1] M. Barry, O. Nakoulima, G. B. Ndiaye, Cauchy System for Parabolic Operator, International Journal of Evolution Equations 8, no. 4 (2013) pp. 277-290.

[2] M. Barry, G. B. Ndiaye, Cauchy system for an hyperbolic operator, Journal of Nonlinear Equations and Applications. Volume 2014, Number 4 pp. 37-52. $\left(\omega_{2,}^{*} \omega_{2}^{*}\right) ;\left(z_{1}^{*}, z_{2}^{*}\right)$ defines respectively in proposition 6 and proposition 8 , we have

$$
\begin{gathered}
J_{2}\left(u_{1}^{*}, u_{2}^{*}, \omega_{1}^{*}, \omega_{2}^{*}, z_{1}^{*}, z_{2}^{*}\right) \leq J_{2}\left(u_{1}, u_{2}, \omega_{1}^{*}, \omega_{2}^{*}, z_{1}^{*}, z_{2}^{*}\right) \\
\leq J_{2}\left(u_{1}, u_{2}, \omega_{1}^{*}, \omega_{2}^{*}, y, y\right)=0
\end{gathered}
$$

From proposition 4 we conclude that

$$
u_{1}^{*}=u_{1}, u_{2}^{*}=u_{2} ; \text { and } z_{1}^{*}=z_{2}^{*}=y
$$

Therefore we have
[3] P. M. Fall, O. Nakoulima, A Sène: On a non-classical boundary values problem for the heat equation. African Diaspora Journal of mathematics (ADJM), Volume 16, Number 2, pp. $59-71$ (2014).

[4] J. P. Kernevez, Enzyme mathematics, North-Holland Publishing Company, 1980.

[5] J. L. Lions. Contrôle optimal de systèmes gouvernés par des équations aux dérivées partielles. Dunod, Gauthier-Villars, Paris 1968.

[6] J. L. Lions, Contrôle des systèmes distribués singuliers. BORDAS, Gauthier-Villars, Paris, 1983 ISBN 2-04-015539-2.

[7] J. L. Lions and E. MAGENES, Problèmes aux limites non homogènes et applications, DUNOD, vol1 Paris, 1968.

[8] J. L. Lions. Perturbations Singulières dans les Problèmes aux Limites et en Contrôle Optimal. Springer-Verlag. Berlin. Heidelberg. New York 1973.

[9] O. Nakoulima, Contrôle de systèmes mal posés de type elliptique, J. Math. Pures et Appl., 1994, 73, 441-453.

[10] O. Nakoulima, G. M. Mophou, Control of Cauchy System for an Elliptic Operator, Acta Mathematica Sinica (English Series) 25, no. 11 (2009) pp. 1819-1834.

[11] J. Smoler, Shock waves, and reaction-diffusion equations, Springer Ver-lag, Berlin-Heidelberg, New York, 1983.

[12] H. V. Stackelberg. Markform undGleichgewicht. Springer, Berlin, Ger-many 1934. 